\title{
Plane Embedding of Dually Contracted Graphs*
}

\author{
Roland Glantz and Walter G. Kropatsch \\ Pattern Recognition and Image Processing Group 183/2 \\ Institute for Computer Aided Automation, Vienna University of Technology \\ Favoritenstr. 9, A-1040 Vienna, Austria phone ++43-(0)1-58801-18358, fax \\ $++43-(0)-1-58801-18392$ \\ $\{\mathrm{glz}, \mathrm{krw}\} @$ prip. tuwien.ac . at
}

\begin{abstract}
The use of plane graphs for the description of image structure and shape representation poses two problems : (1) how to obtain the set of vertices, the set of edges and the incidence relation of the graph, and (2) how to embed the graph into the plane image. Initially, the image is represented by an embedded graph $G$ in a straight forward manner, i.e. the edges of $G$ represent the 4 -connectivity of the pixels. Let $\bar{G}$ denote a (planar) abstract dual of $G$. Dual graph contraction is used to reduce the pair $(\bar{G}, G)$ to a pair $(\bar{H}, H)$ of planar abstract duals. Dual graph contraction is unsymmetric due to an extra condition on the choice of the contraction kernels in $G$. This condition is shown to be necessary and sufficient for $H$ to be embedded onto $G$. The embedding is applied to the description of image structure and to shape representation.
\end{abstract}

\section{Introduction}

A key concept in combinatorial topology is the separation of topology (the graph) and embedding (of the graph) [Fra96]. In a hierarchical representation of an image by a sequence of plane graphs on increasing levels of abstraction and scale, however, the hierarchy should also be reflected by the embedding. Thus, the embedding of a lower level graph induces constraints on the embedding of higher level graphs. Let a high and a low level graph be denoted by $H=\left(V_{H}, E_{H}\right)$ and $L=\left(V_{L}, E_{L}\right)$ respectively. The hierarchy is reflected by the embedding of $H$ and $L$ (Fig. 1), if

1. $V_{H} \subset V_{L}$,

2. each embedded edge from $E_{H}$ (as subset of the plane) is a union of embedded edges from $E_{L}$,

3. each region from the embedding of $H$ (as subset of the plane) is a union of regions from the embedding of $L$.

Note the transitivity of the above conditions. The high level graph is a connected topological minor of the low level graph [Die97]. This paper is devoted to

\footnotetext{
* This work has been supported by the Austrian Science Fund (FWF) under grant S7002-MAT and grant P14445-MAT.
} 
hierarchies of graphs as obtained by dual graph contraction [KM95]. It will be shown that an embedding of a higher level graph may always be derived from the embedding of a lower level graph such that the above conditions are fulfilled. Note, however, that dual graph contraction yields two hierarchies of graphs: on a planar graph and on its (planar) abstract dual. It will turn out that, in general, it is impossible to obtain a proper embedding of both hierarchies. Proper embedding of graph hierarchies is crucial for document image analysis [BK99] and the description of image structure [GEK99]. In this paper we propose a new application for dual graph contraction in which embedding is crucial, i.e. shape representation. The outline of the paper is as follows: In Section 2 dual graph

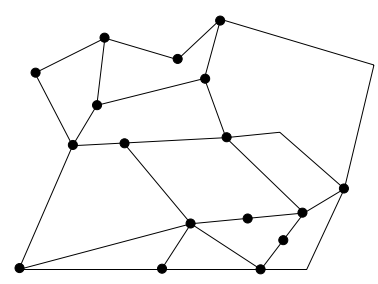

(a)

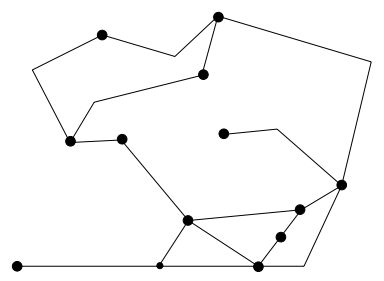

(b)

Fig. 1. Hierarchical embedding. (a) Low level. (b) High level

contraction is described in terms of equivalence relations. In Section 3 the embedding of high level graphs onto low level graphs is related to the unsymmetry of dual graph contraction. Section 4 demonstrates the use of the embedding for the description of image structure. Section 5 is devoted to shape representation by means of embedded graphs that describe the structure of distance transforms. We conclude in Section 6.

\section{Dual Graph Contraction by Equivalence Relations}

Throughout the paper we refer to the following definition of a graph.

Definition 1 (Graph). A graph $G=(V, E)$ is given by a finite set $V$ of elements called vertices, a finite set $E$ of elements called edges with $E \cap V=\emptyset$ and an incidence relation $\iota$ which associates with each edge $e \in E$ a subset of $V$ with one or two elements. The vertices in $\iota(e)$ are called the end vertices of $e$.

Note that the definition includes graphs with self-loops (i.e. edges with only one end vertex) and multiple edges (i.e. several edges with identical sets of end vertices). 
Let $(\bar{G}, G)$ denote a pair of planar graphs, where $\bar{G}$ is an abstract dual [Die97] of $G$. We write $\bar{G}=(\bar{V}, \bar{E})$ and $G=(V, E)$. Dual edge contraction [Kro95] [KM95] of the pair $(\bar{G}, G)$ is specified by contraction kernels which form a spanning forest $\bar{F}$ of $\bar{G}$. The result of dual edge contraction consists of a pair denoted by $(\bar{G} / \bar{F}, G \backslash F)$, in which

- $\bar{G} / \bar{F}$ is obtained from $\bar{G}$ by contracting the edges of $\bar{F}$ as shown below.

- $F$ denotes the subgraph of $G$ that is induced by the edges which are dual to the edges of $\bar{F}$.

- $G \backslash F$ is obtained from $G$ by the removal of all edges from $F$.

In the following, the vertex set, the edge set and the incidence relation of $\bar{G} / \bar{F}$ are defined by means of an equivalence relation on the vertex set $\bar{V}$ of $\bar{G}$.

The spanning forest $\bar{F}$ partitions $\bar{V}$ : each vertex belongs to exactly one connected component of $\bar{F}$. Hence, the binary relation $\sim_{\bar{F}}$ defined as

$$
\bar{v} \sim \bar{F} \bar{w}: \Leftrightarrow \bar{v} \text { and } \bar{w} \text { belong to the same connected component of } \bar{F}
$$

is an equivalence relation on $\bar{V}$. Let the equivalence class of $\bar{v}$ be denoted by $[\bar{v}]_{\bar{F}}$. The vertex set of $\bar{G} / \bar{F}$ equals the quotient of $\bar{V}$ by $\sim_{\bar{F}}$, defined as

$$
\bar{V} \backslash \sim_{\bar{F}}:=\left\{[\bar{v}]_{\bar{F}} \mid \bar{v} \in \bar{V}\right\} .
$$

If $\bar{E}_{\bar{F}}$ denotes the set of edges in $\bar{F}$, the edge set of $\bar{G} / \bar{F}$ equals $\bar{E} \backslash \bar{E}_{\bar{F}}$. The incidence relation $\iota_{\bar{G}} / \bar{F}$ of $\bar{G} / \bar{F}$ is derived from the incidence relation $\iota_{\bar{G}}$ of $\bar{G}$ in the following way: For a non-loop $\bar{e} \in \bar{E} \backslash \bar{E}_{\bar{F}}$ with $\iota_{\bar{G}}(\bar{e})=\{\bar{v}, \bar{w}\}$ set

$$
\iota_{\bar{G}} / \bar{F}(\bar{e}):=\left\{[\bar{v}]_{\bar{F}}\right\} \cup\{[\bar{w}] \bar{F}\} .
$$

For a loop $\bar{e} \in \bar{E} \backslash \bar{E}_{\bar{F}}$ with $\iota_{\bar{G}}(\bar{e})=\{\bar{v}\}$ set

$$
\iota \bar{G} / \bar{F}(\bar{e}):=\left\{[\bar{v}]_{\bar{F}}\right\} .
$$

The second step of dual graph contraction, i.e dual face contraction, consists in the contraction of edges from the graph $G \backslash F$ and the removal of the corresponding dual edges in $\bar{G} / \bar{F}$. The contraction kernels in $G \backslash F$ are to form a spanning forest $F^{\prime}$ of $G \backslash F$. However, each connected component of $F^{\prime}$ is required to contain at most one vertex whose degree in $G \backslash F$ is larger than two. In the following, this constraint will be referred to as degree constraint. It assures that the removal of the corresponding dual edges in $\bar{G} / \bar{F}$ is restricted to so called redundant edges, i.e. parallel edges, or loops that do not surround a subgraph of $\bar{G} / \bar{F}$ (Fig. 2).

The result of dual graph contraction is a pair $(\bar{H}, H)$ of planar graphs, in which $\bar{H}$ is an abstract dual of $H$. If $\overline{F^{\prime}}$ denotes the subgraph of $\bar{G}$ that is induced by the edges which are dual to the edges in $F^{\prime}$, then

$$
H=(G \backslash F) / F^{\prime} \quad \text { and } \quad \bar{H}=(\bar{G} / \bar{F}) \backslash \overline{F^{\prime}} .
$$




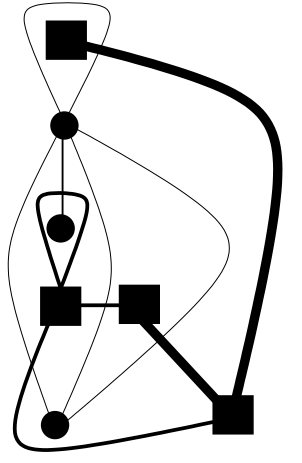

(a)

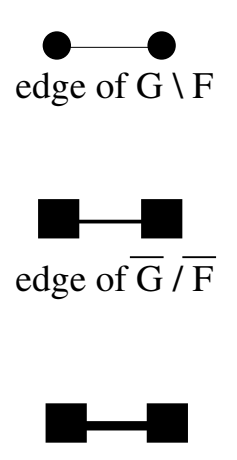

edge of kernel

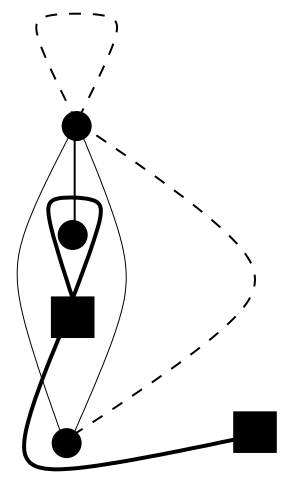

(b)

Fig. 2. Dual face contraction. (a) Contraction kernel in $G \backslash F$. (b) Removal of redundant edges in $\bar{G} / \bar{F}$ (dotted)

The degree constraint ensures that the degree of a vertex $[v]_{F^{\prime}}$ in $H$ cannot exceed the degree of vertex $v$ in $G \backslash F$. Furthermore, the degree of vertex $v$ in $G \backslash F$ is restricted by the degree of the vertex $v$ in $G$. Hence, if the degree of vertex $[v]_{F^{\prime}}$ in $H$ is denoted by $\operatorname{deg}_{H}\left([v]_{F^{\prime}}\right)$, the following inequation holds:

$$
\operatorname{deg}_{H}\left([v]_{F^{\prime}}\right) \leq \operatorname{deg}_{G \backslash F}(v) \leq \operatorname{deg}_{G}(v) \quad \forall v \in V .
$$

In particular, the maximal vertex degree in $H$ is restricted by the maximal vertex degree in $G$.

\section{Embedding of Contracted Graphs}

Let $G=(V, E)$ denote a plane graph and assume $F$ to be a spanning forest of $G$ that fulfills the degree constraint. An embedding of $G / F$ can be obtained from the embedding of $G$ in the following way (Fig. 3):

1. Interpret the connected components of $F$ as rooted trees: If the connected component has a (unique) vertex $r$, whose degree in $\mathbf{G}$ is larger than two, declare $r$ to be the root. Otherwise declare any vertex of the connected component to be the root.

2. Let $R$ denote the set of all roots from step 1 . For the vertex set of $G / F$, i.e. the quotient $V \backslash \sim_{F}$ of $V$ by $\sim_{F}$ the following holds

$$
V \backslash \sim_{F}=\left\{[r]_{F} \mid r \in R\right\} .
$$

For each $r \in R$ set the location of $[r]_{F}$ to the location of $r$ in $G$. 


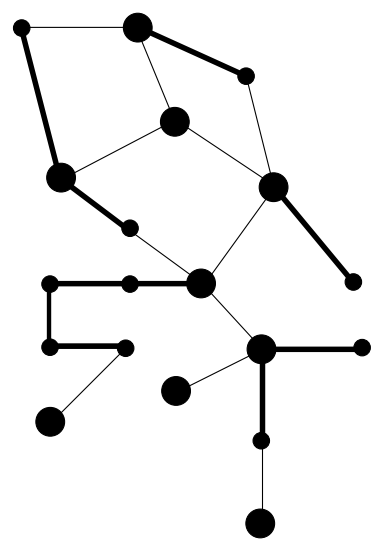

(a)

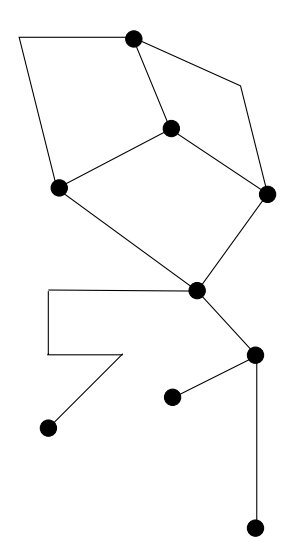

(b)

Fig. 3. (a) Graph $G$. Bold edges belong to spanning forest $F$. $F$ fulfills the degree constraint. Roots are enlarged. (b) Embedding of $G / F$

3. Let $E_{F}$ denote the set of edges in $F$. The edge set of $G / F$ equals $E \backslash E_{F}$. Let $e$ be an edge from $E \backslash E_{F}$ and let the connected components of $F$, where the end vertices of $e$ in $\mathbf{G}$ belong to, be denoted by $C C_{1}$ and $C C_{2}$. Since the roots $r_{1}$ and $r_{2}$ of $C C_{1}$ and $C C_{2}$ are the only vertices in $C C_{1} \cup C C_{2}$ that may have a degree greater than two in $\mathbf{G}$, there is a unique path $\Pi=\Pi(e)$ from $r_{1}$ to $r_{2}$ in $G$ which contains $e$. Furthermore, for any two edges $e, e^{\prime}$ the paths $\Pi(e)$ and $\Pi\left(e^{\prime}\right)$ may only intersect at vertices from $R$. The embedding of $e$ in $\mathbf{G} / \mathbf{F}$ is given by the union of all (embedded) edges that belong to $\Pi(e)$.

Note that the embedding of the paths, as described in step 3, fails whenever $F$ does not fulfill the degree constraint. Hence, the degree constraint is necessary and sufficient for the graph $G / F$ to be embedded onto $G$.

\section{Image Structure}

Gray level images may be interpreted as digital elevation models [DEM], in which the altitudes are given by the gray levels. In [KD94] the structure of an image is defined via the crest lines of the corresponding DEM. In this section, a plane graph is constructed, which describes the crest lines in a DEM. It is referred to as crest graph. The crest graph for the gray level image in Fig. 4(a) is depicted in Fig. 5(a). In contrast to the graph constructed in [GEK99], the crest graph has the following properties:

- The crest graph is embedded such that the edges describe the run of the crest lines in the DEM. 
- The vertex set of the crest graph may include vertices that do not represent a hill of the DEM. The extra vertices represent branching points of crest lines that are not located on the top of hills.

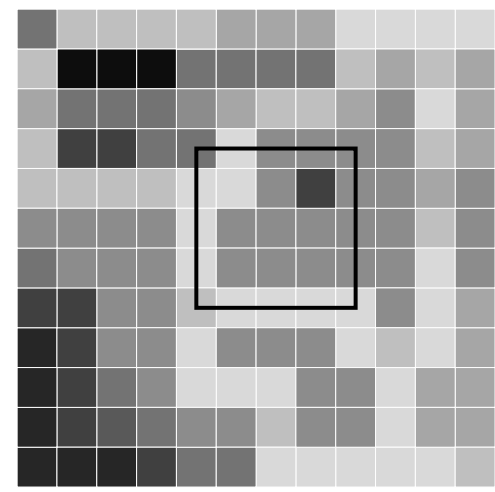

(a)
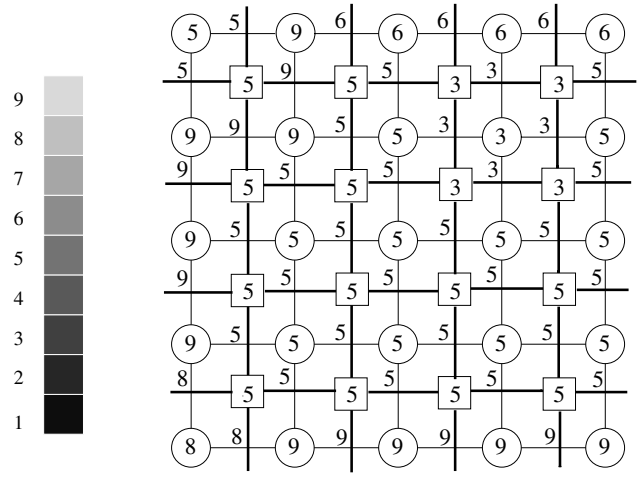

(b)

Fig. 4. (a) The gray levels of the pixels. (b) The pair $(\bar{G}, G)$ restricted to the square region marked in (a). The vertices of $\bar{G}[\mathrm{G}]$ are depicted as squares [circles]

The crest graph is constructed in the following way:

1. The DEM is transformed into a pair $(\bar{G}, G)$ of attributed plane graphs, in which $G$ and $\bar{G}$ are plane duals [Die97]. The pair $(\bar{G}, G)$ will later be transformed into a pair $\left(\overline{G_{c}}, G_{c}\right)$, in which $G_{c}$ is the crest graph. An example of $(\bar{G}, G)$ is given in Fig. 4(b). The vertices of $G$ represent the pixels centers, while the edges of $G$ indicate the 4 -neighborhood of the pixels. The vertex value is set to the altitude associated with the corresponding pixel. Throughout the transformation of $(\bar{G}, G)$ into $\left(\overline{G_{c}}, G_{c}\right)$, the value of an edge from $G$ is to indicate the lowest altitudes along the embedded edge. Hence, the value of an edge $e$ with end vertices $u$ and $v$ is initialized to the minimum of the vertex values of $u$ and $v$. The vertices of $\bar{G}$ represent the regions of $G$ and are located at the crossings of the pixel borders. The edges of $\bar{G}$ are straight line segments that reflect the 4-neighborhood of the regions from $G$. Their values are initialized to the values of the corresponding dual edges in $G$. A vertex $\bar{v}$ of $\bar{G}$ is initialized to the minimal value of all edges to surround the region (basin) represented by $\bar{v}$, i.e. the minimal value of all edges in $\bar{G}$ that are incident to $\bar{v}$. Throughout the construction of $\left(\overline{G_{c}}, G_{c}\right)$ from $(\bar{G}, G)$, the value of a vertex $\bar{v}$ from $\bar{G}$ is to indicate the lowest altitude in the region (basin) represented by $\bar{v}$. 


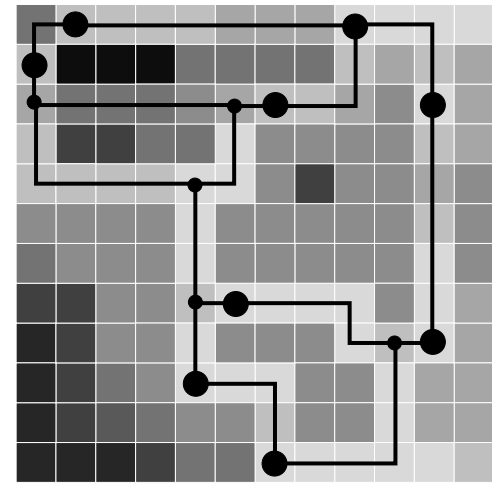

(a) Crest graph

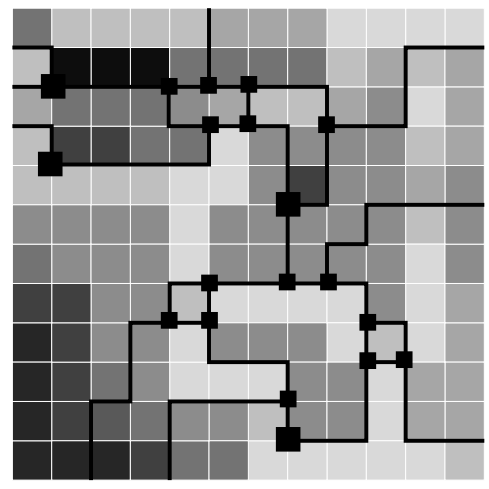

(b) River line graph

Fig. 5. The crest graph and the river line graph. The vertices which represent hills respectively basins are drawn larger

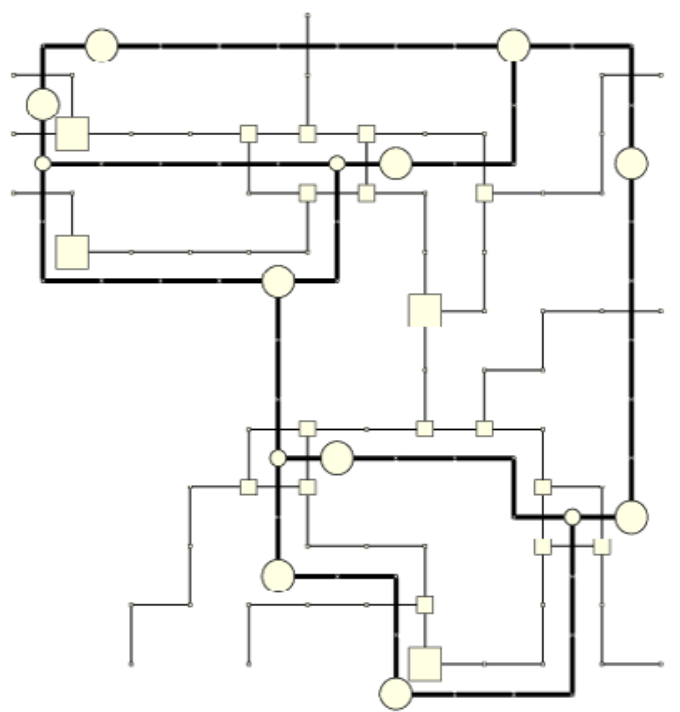

Fig. 6. Overlay of the crest graph (thick lines) and the river line graph (thin lines) from Fig. 5 
2. The pair $\left(\overline{G_{c}}, G_{c}\right)$ is generated from $(\bar{G}, G)$ by a sequence of monotonic dual graph contractions [GEK99]: Besides the general requirements described in Section 2, each contraction kernel of $G[\bar{G}]$ has to contain exactly one local maximum [minimum] of vertex values. The vertex value in the contracted graph is set to the maximum [minimum] of the vertex values in the corresponding contraction kernel. Monotonic dual graph contraction stops when no contraction kernel with more than one vertex can be chosen.

3. The crest graph $G_{c}$ is embedded into the plane as described in Section 3.

If monotonic dual graph contraction is applied to the pair $(G, \bar{G})$ instead of $(\bar{G}, G)$, the graph $\bar{G}$ will be transformed into a graph that describes the river lines of a DEM. The river line graph of Fig. 4(a) is depicted in Figure 5(b)). An overlay of the the crest graph and the river line graph is shown in Fig. 6. Note that, in general, the crest graph and the river line graph are not even abstract duals. However, embedding the two graphs into the DEM, two basins separated by a crest line are always connected by at least one river line that crosses the crest line at a local minimum (saddle point).

The calculation of the crest graph was implemented in $\mathrm{C}^{++}$based on LEDA [MN99]. In order to describe gray level edges in images, we calculated the crest graph on the image transformed by the Sobel operator (Fig. 7). Note that the crest graph is always connected.

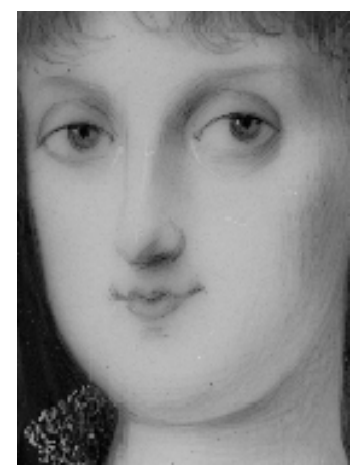

(a)

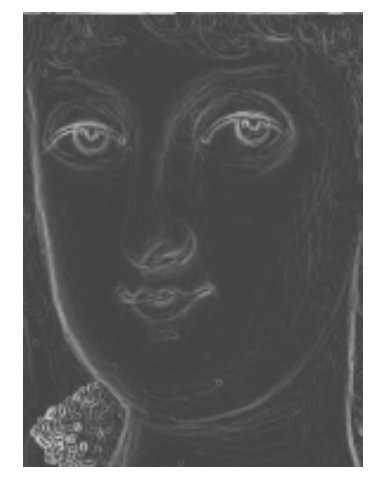

(b)

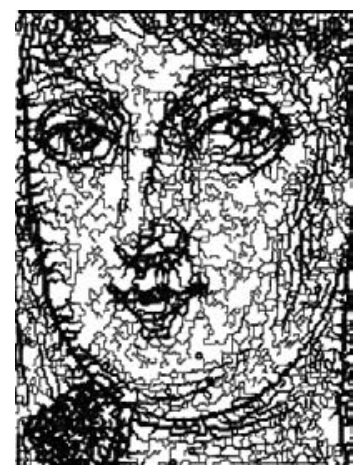

(c)

Fig. 7. (a) Gray level image. (b) Input image for the calculation of the crest graph (Sobel operator on (a)). (c) Crest graph of (b). The widths of the edges correspond to the edge values, i.e. the altitudes of the saddle points 


\section{Shape from the Structure of Distance Transforms}

In this section the shape of a 4-connected set $S$ of square pixels is represented by an embedded graph. The embedded graph is the crest graph calculated from a distance transform of $S$, i.e. the gray values indicate the distance of the pixel to the outside of $S$. The calculation of the crest graph is done exactly as explained in the previous section. Note that this concept is not restricted to a special grid or to a special distance.

As an example consider the 4-connected set of pixels in Fig. 8(b). The numbers in Fig. 8(b) indicate the chamfer-3-4 distance [CM91] from the pixels to the outside. A sphere with respect to the chamfer-3-4 distance is shown in Fig. 8(a). The crest graph of the distance transform is depicted in Fig. 8(c). The advantages

\begin{tabular}{|c|c|c|c|c|}
\hline & 7 & 6 & 7 & \\
\hline 7 & 4 & 3 & 4 & 7 \\
\hline 6 & 3 & & 3 & 6 \\
\hline 7 & 4 & 3 & 4 & 7 \\
\hline & 7 & 6 & 7 & \\
\hline
\end{tabular}

(a)

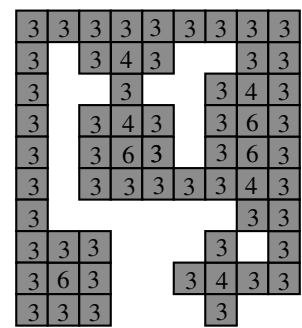

(b)

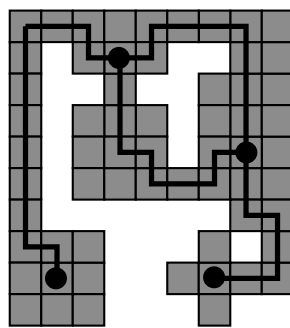

(c)

Fig. 8. (a) Sphere with respect to the chamfer-3-4 distance. (b) Chamfer-3-4 distance transform of an object. (c) Crest graph of (b)

of the crest graph compared to the medial axis [Ser82] are as follows.

- Whatever structuring elements are taken for the computation of the distance transform, the crest graph is always connected.

- No pruning is needed [Ogn94].

- The structure of the crest graph, i.e. the crest graph without its embedding, yields a more compact and more abstract description of the shape.

In contrast to the medial axis, shape representation by means of the crest graph is lossy. In particular, the crest graph does not represent narrowing dead ends. In the future we will try to overcome this drawback by a modification of the distance transform: the pixel values at the edge of the image are raised such that they form a new summit. The edges which connect this summit with the summits in the inner part of the image will run through the narrowing dead ends. 


\section{Conclusion}

The hierarchy of planar graphs, as obtained by dual graph contraction, has been combined with a hierarchical plane embedding of the graphs. It was shown that, in general, it is impossible to find such an embedding also for the corresponding hierarchy of the dual graphs. The proposed hierarchical embedding is very useful for the description of image structure by monotonic dual graph contraction and improves the method proposed in [GEK99]. If the image structure is computed on gray values coming from a distance transform of a binary image, monotonic dual graph contraction yields a skeleton-like plane graph. In contrast to the skeletons formed by pixels no pruning is necessary. Future work will focus on the proper representation of narrowing dead ends.

\section{References}

BK99. Mark J. Burge and Walter G. Kropatsch. A minimal line property preserving representation of line images. Computing, 62:355 - 368, 1999. 349

CM91. Jean Marc Chassery and Annick Montanvert. Géometrie Discrète en Imagery. Hermes, Paris, 1991. 356

Die97. Reinhard Diestel. Graph Theory. Springer, New York, 1997. 348, 350, 353

Fra96. Jean Francon. On Recent Trends in Discrete Geometry in Computer Science. In Serge Miguet, Michel Montanvert Annick, and Stéphane Ubéda, editors, Discrete Geometry for Computer Imagery, DGCI'96, volume Vol. 1176 of Lecture Notes in Computer Science, pages 3-16, Lyon, France, 1996. Springer, Berlin Heidelberg, New York. 348

GEK99. Roland Glantz, Roman Englert, and Walter G. Kropatsch. Dual image graph contractions invariant to monotonic transformations of image intensity. In Proc. of the 2nd IAPR Workshop on Graph-based Representation, 1999. 349, $352,355,357$

KD94. J. Koenderink and A. van Doorn. Image structure. In E. Paulus and F. Wahl, editors, Mustererkennung 1997, pages 401-408. Springer, 1994. 352

KM95. Walter G. Kropatsch and Herwig Macho. Finding the structure of connected components using dual irregular pyramids. In Cinquième Colloque $D G C I$, pages 147-158. LLAIC1, Université d'Auvergne, ISBN 2-87663-040-0, September 1995. 349, 350

Kro95. Walter G. Kropatsch. Building Irregular Pyramids by Dual Graph Contraction. IEE-Proc. Vision, Image and Signal Processing, 142(6):366 - 374, 1995. 350

MN99. K. Mehlhorn and S. Näher. The LEDA Platform of Combinatorial and Geometric Computing. Cambridge University Press, Cambridge, U. K., 1999. 355

Ogn94. R. L. Ogniewicz. A Multiscale MAT from Voronoi Diagrams: The SkeletonSpace and its Application to Shape Description and Decomposition. In Carlo Arcelli, Luigi P. Cordella, and Gabriella Sanniti di Baja, editors, 2nd Intl. Workshop on Visual Form, pages 430-439, Capri, Italy, June 1994. 356

Ser82. Jean Serra. Image and Mathematical Morphology, volume 1. Academic Press, London, G. B., 1982. 356 